\section{The PAZOREAL noninterventional study to assess effectiveness and safety of pazopanib and everolimus in the changing metastatic renal cell carcinoma treatment landscape}

\author{
Peter J Goebell ${ }^{*, 1}$, Christian Doehn², Carsten Grüllich³, Viktor Grünwald ${ }^{4}$, \\ Thomas Steiner ${ }^{5}$, Rainer Ehneß ${ }^{6}$ \& Manfred Welslau ${ }^{7}$
}

VEGFR and mTOR inhibitors are broadly used in metastatic renal cell carcinoma (mRCC) therapy, and sequential first-line pazopanib (VEGFR inhibitor) and second-line everolimus (mTOR inhibitor) is a standard treatment option. Nivolumab and lenvatinib/everolimus combination was recently approved in Europe for use in mRCC after previous therapy. Prospective routine data on sequential therapy including nivolumab and/or lenvatinib are missing. This is a prospective, noninterventional study, which evaluates the effectiveness, tolerability, safety and quality of life following 450 patients with mRCC starting either on pazopanib as first-line therapy or third-line everolimus plus/minus lenvatinib following nivolumab. Adults with histologically confirmed mRCC of any subtype, who have a life expectancy of at least 6 months, are eligible.

First draft submitted: 21 February 2017; Accepted for publication: 10 April 2017; Published online: 19 May 2017

Renal cell carcinoma (RCC) is diagnosed in about 15,500 patients per year in Germany [1]. Currently, one of the standard treatment options for patients with metastatic RCC (mRCC) is sequential firstline pazopanib, a VEGFR inhibitor followed by everolimus, an mTOR inhibitor, as second-line treatment [2-4]. Phase III studies demonstrated that the median progression-free survival (PFS) was significantly longer for patients who received pazopanib over placebo in the first line ( $9.2 \mathrm{vs}$ 4.2 months; $p<0.001$ ) [5] and for patients who received everolimus over placebo in the second-or later-line setting ( 4.9 vs 1.9 months; $\mathrm{p}<0.001$ ) [6]. Moreover, pazopanib has been shown not to be inferior to sunitinib in a large Phase III trial [3,7].

The treatment landscape for patients with $\mathrm{mRCC}$ has changed considerably in recent years. The Phase III CheckMate-025 trial evaluated nivolumab, a programmed death-1 immune checkpoint inhibitor, and demonstrated that it led to a survival advantage over everolimus in

'Department of Urology, University Erlangen, Erlangen, Germany

2Urologikum Lübeck, Lübeck, Germany

${ }^{3}$ Department of Medical Oncology at National Center for Tumor Diseases (NCT), University Hospital Heidelberg, Heidelberg, Germany ${ }^{4}$ Department of Hematology, Hemostaseology, Oncology \& Stem Cell Transplantation, Medical School of Hannover, Hannover,

\section{Germany}

${ }^{5}$ Department of Urology, HELIOS Klinikum Erfurt, Erfurt, Germany

${ }^{6}$ Novartis Pharma GmbH, Nuernberg, Germany

${ }^{7}$ Haemato-Onkologische Schwerpunktpraxis am Klinikum Aschaffenburg, Aschaffenburg, Germany

*Author for correspondence: Tel.: +49 09131822 3122; Fax: +49 09131822 5061; peter.goebell@uk-erlangen.de

\section{KEYWORDS}

- Afinitor • everolimus

- metastatic renal cell carcinoma $\bullet \mathrm{mRCC}$

- nivolumab

- noninterventional

- Opdivo • pazopanib

- PAZOREAL • Votrient 
previously treated patients with mRCC (median overall survival [OS]: 25.0 vs 19.6 months; $\mathrm{p}=$ 0.002) [8]. Based on these results, nivolumab was approved in Europe in April 2016 as monotherapy for patients with $\mathrm{mRCC}$ after they have received prior therapy [9]. Furthermore, EMA granted marketing approval for the c-MET and VEGFR2 inhibitor, cabozantinib, and the multikinase inhibitor lenvatinib in combination with everolimus, for the treatment of mRCC patients in 2016 [10,11]. In the METEOR pivotal study, cabozantinib has shown to prolong median PFS significantly as compared with everolimus (7.4 months vs 3.8 months; $\mathrm{p}<$ 0.001) $[12,13]$. The combination of lenvatinib plus everolimus demonstrated a significantly longer median OS as compared with everolimus monotherapy in the pivotal Phase II trial (25.5 vs 15.4 months; $p=0.024)$ [14]. The recently updated international guidelines on RCC have already included nivolumab and cabozantinib into their recommendations $[3,4]$.
Furthermore, with the upcoming amendment to the German S3 guideline on RCC, cabozantinib and nivolumab are expected to be defined as standard in the second-line therapy of RCC. Axitinib, everolimus and lenvatinib plus everolimus are options in this setting and the latter combination is likely to be entered into the amended guideline as well [15-17].

\section{The PAZOREAL study}

The noninterventional PAZOREAL (Pazopanib and Everolimus in a Real-world Setting) study is designed to observe the real-world use of pazopanib, everolimus (with or without lenvatinib) or nivolumab given in first line through third-line therapy for patients with mRCC. This study is funded by Novartis Pharma GmbH, Nuernberg, Germany.

\section{Background \& rationale}

Although clinical trials provide essential information on efficacy and tolerability, they do not

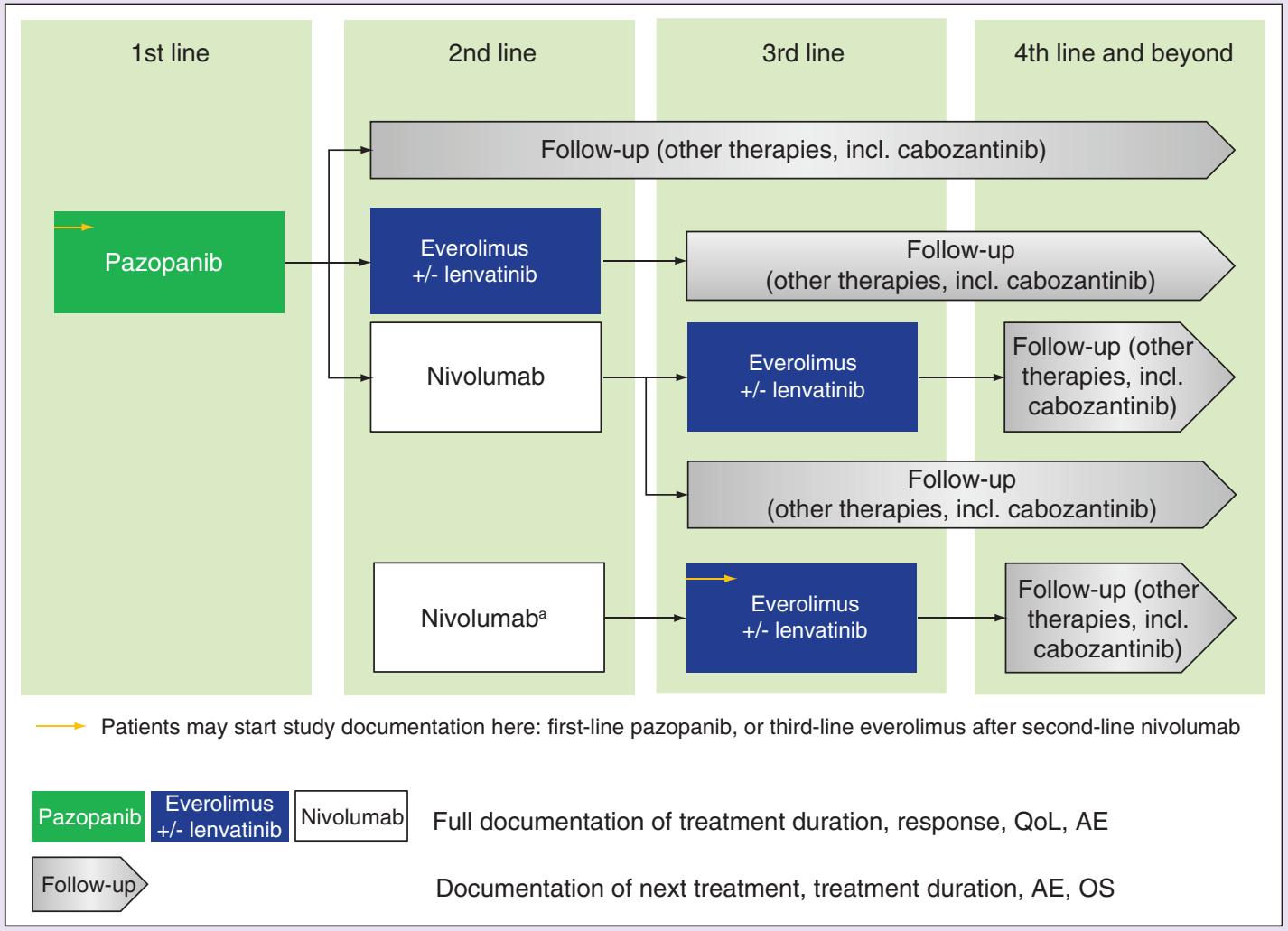

Figure 1. Study design.

${ }^{\dagger}$ Retrospective documentation of nivolumab in the second line.

AE: Adverse event; mRCC: Metastatic renal cell carcinoma; OS: Overall survival; QoL: Quality of life. 
Box 1. Inclusion and exclusion criteria.

\section{Inclusion criteria}

- Histologically confirmed mRCC of any histology

- Life expectancy of at least 6 months

- Systemic treatment that either starts with first-line pazopanib or continues with third-line everolimus after second-line nivolumab

- Written informed consent

- Planned treatment is in line with the respective current German SmPC

\section{Exclusion criteria}

- $<18$ years of age

- Unable to provide written informed consent

- Contraindication according to the respective current German SmPC

- Active treatment in a research study

- Parallel documentation in another registry or follow-up of a clinical trial is allowed

Retrospective documentation of a given line of treatment is possible if treatment has started a maximum of 8 weeks prior to the patient's written informed consent.

mRCC: Metastatic renal cell carcinoma; SmPC: Summary of product characteristics.

mirror the everyday clinical use of agents used to treat patients with mRCC. A major handicap of clinical trials is the (indispensable) limitation of the patient population via inclusion and exclusion criteria. A comparison of data from clinical trials and from real-world studies suggests that patients in routine practice differ from patients treated in clinical trials and that almost $60 \%$ of mRCC patients in German routine practice would be ineligible for participation in clinical trials [18].

Real-world data are limited for first-line pazopanib, and even more limited for sequential pazopanib and everolimus (or another targeted agent) in patients with mRCC. An ongoing prospective $\mathrm{mRCC}$ clinical registry is investigating real-life treatment outcomes with systemic treatment in all therapy lines. However, relevant data on drugs which became available in the recent few years, such as pazopanib and especially the recently approved drugs nivolumab, cabozantinib and lenvatinib are still lacking $[18,19]$. Moreover, the approval of nivolumab has influenced the therapy algorithms for patients with mRCC to a great extent. The two recently approved agents, cabozantinib and lenvatinib in combination with everolimus additionally augment the treatment landscape. Data on the daily clinical use are needed to further evaluate the safety, tolerability, effectiveness and quality of life (QoL) of the various available therapy sequences in mRCC.

Hence, the noninterventional PAZOREAL study was designed to observe how patients are treated with first-line pazopanib, and which therapies are subsequently used in the second-and third-line settings with regard to the evolving treatment landscape. At the time this study was designed, nivolumab had not been approved in the EU, but based on published study results, the timely approval of nivolumab was assumed. Thus, the study design allows in-label nivolumab documentation since the time the drug has gained EMA approval for the treatment of mRCC. Furthermore, PAZOREAL provides real-world data of the evolving landscape of RCC, including limited efficacy data on the new agents, cabozantinib and lenvatinib.

\section{Design}

\section{- Study design}

PAZOREAL is a prospective, noninterventional study that will evaluate the effectiveness, tolerability, safety and QoL in patients with mRCC treated with first-line pazopanib, second-line nivolumab or everolimus or thirdline everolimus after nivolumab. Other second- and later-line in-label treatments are also documented. The documentation of an eligible patient can either start in the first line, if pazopanib has been chosen to be the first-line treatment, or in the third line, if it was decided to treat the patient with everolimus (Figure 1). Patients whose treatment with first-line pazopanib or third-line everolimus has started within 8 weeks prior to written informed consent can be included (i.e., a maximum of 8 weeks is allowed for retrospective documentation). A full documentation is required 


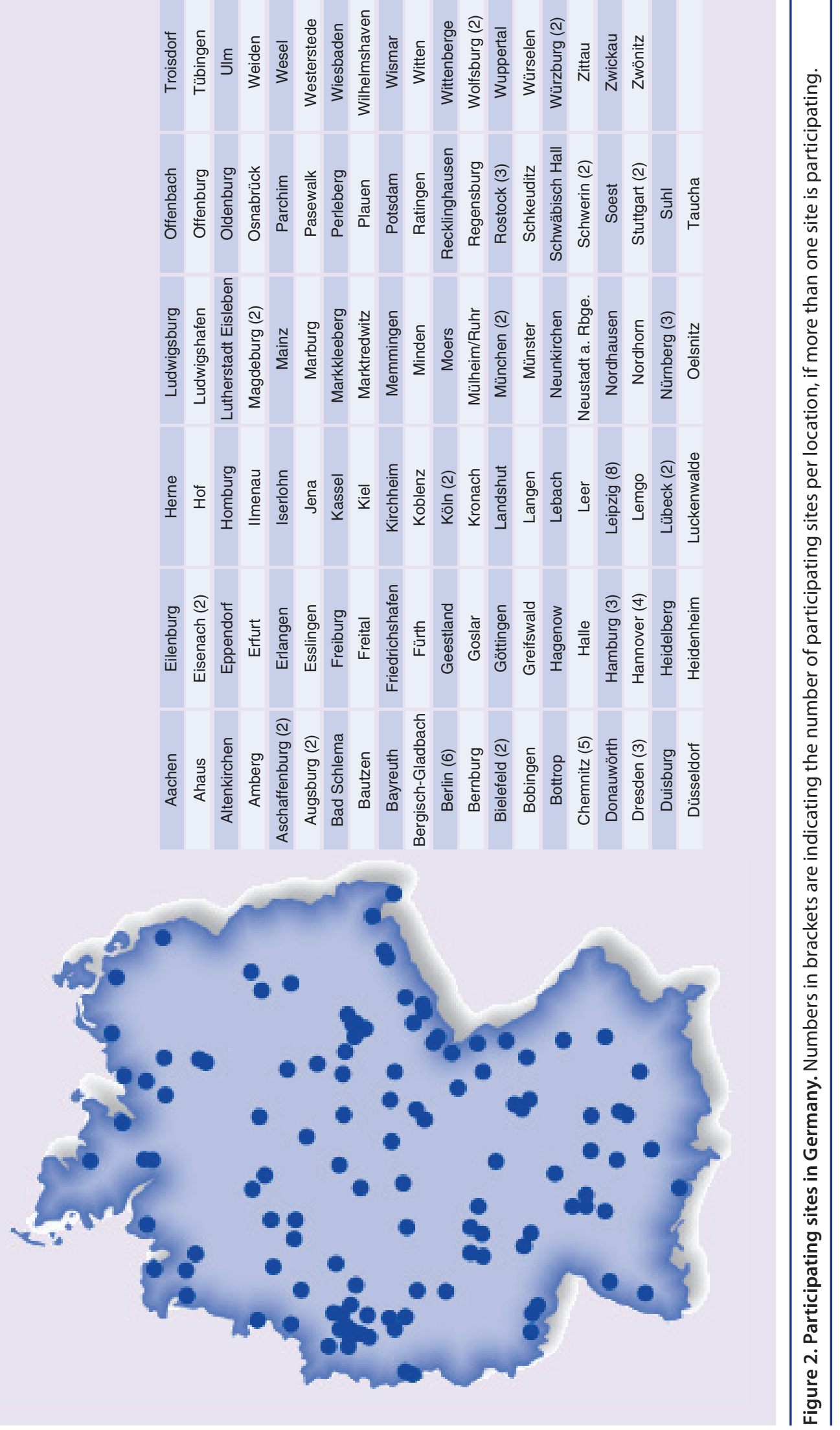


Table 1. Planned milestones.

\begin{tabular}{|ll|}
\hline Study start (first patient in): & December 2015 \\
\hline Recruitment end (last patient in): & Q1 2018 \\
\hline End of observation period: & Q2 2021 \\
\hline Study end (end of data collection): & Q3 2021 \\
\hline Study duration: & $\begin{array}{l}\text { An average treatment duration of 18 months is assumed; } \\
\text { additional 6-24 months of follow-up for OS }\end{array}$ \\
\hline Completion of study report: & Q2 2022 \\
\hline Primary publication date: & Q4 2022 \\
\hline Q: Quarter; OS: Overall survival. & \\
\hline
\end{tabular}

for patients treated with first-line pazopanib, second-line nivolumab or everolimus following pazopanib or third-line everolimus (following nivolumab in the second line). The full documentation comprises patients' history; treatment and dosing parameters (dose reduction/ interruption rates and reasons); disease assessments (time on drug for all lines of therapy); adverse events (AEs) with a focus on the effect of lab analyses frequency on incidence and severity of AEs with particular emphasis on liver AEs, evaluation of AEs with respect to concomitant medications and discontinuation of treatment due to toxicity; follow-up treatments; and OS. For other treatment regimens in the second-and later-line setting, a reduced documentation is applied ('Follow-Up' documentation), including information on the substance and the duration of the chosen treatment, survival, AEs and disease progression (yes/no), among others.

Provided that a patient has given written informed consent to participate in the QoL assessments and if these assessments are in accordance with the institution's daily practice, standardized EQ-5D-5L questionnaires can be completed by the patient.

Immediately after nivolumab was approved for the treatment of mRCC in Europe, the study was opened for nivolumab documentation in the second line (Figure 1). For patients whose documentation started with first-line pazopanib and continues with nivolumab in the second line, a full documentation is required. For patients entering PAZOREAL in the third line after second-line nivolumab, the preceding nivolumab therapy is documented retrospectively.

Patients already participating in PAZOREAL and receiving in-label cabozantinib in the second- or later-line setting are documented as 'Follow-Up' (reduced documentation, see above). Depending on the frequency of use observed during the inclusion period of the study, a separate 'arm' for in-label cabozantinib in second- or later-line treatment may be introduced via an amendment to the observation plan. For patients receiving in-label lenvatinib plus everolimus in the second line after first-line pazopanib or in the third line, a full documentation of treatment is possible in the current setting.

\section{- Eligibility criteria}

Documentation of a patient is only allowed if drug administration is in accordance with the respective Summary of Product Characteristics, and if the patient has provided written informed consent prior to documentation start. The decision to treat the patient with the respective drug must have been made independently and prior to start-of-study documentation. Adults with a histologically confirmed diagnosis of mRCC of any histological subtype, who have a life expectancy of at least 6 months and whose systemic treatment will either start with first-line pazopanib or continue with third-line everolimus after second-line nivolumab, are eligible for documentation (Box 1).

\section{- Planned sample size}

This observational study is conducted in more than 150 German sites (hematologic/oncologic and urologic private practices as well as hospitals) with a planned total number of 450 patients (Figure 2). Due to the noninterventional character of this study, no formal sample size calculation was conducted. However, this sample size was chosen to guarantee representativeness for the investigated population. Four planned interim analyses (IAs) distributed evenly over the study duration will allow for potential adjustments 
of sample size and/or study design during the course of the study if relevant trends are detected, for instance with regard to subgroup analyses.

\section{- Planned study period \& current status}

The first patient was accrued in December 2015. As of 31 March 2017, 310 patients have been documented within the scope of this noninterventional study, all patients started first-line therapy with pazopanib (Figure 3). Following first-line pazopanib, 39 patients were subsequently treated with nivolumab and 4 patients received subsequent everolimus. The fast collection of individual datasets collected in the depicted period of time mirrors the high interest in the execution of this project and also implements the growing interest of the community to gain insight into routine use of new therapies and their sequential application.

It is expected that recruitment of new patients will be stopped in the first quarter of 2018, as soon as the planned number of 450 patients has been reached. Data collection will be continued for approximately 3 years after recruitment stops. Further milestones are summarized in Table 1.

\section{- Study procedures}

As this is a noninterventional study, no interventions or specific time points for patient visits, disease assessments or other investigations are stipulated.

\section{- Objectives}

The primary objective is to evaluate the effectiveness of first-line pazopanib and second-line and third-line everolimus in the treatment of patients with mRCC in a real-world setting, by measuring the duration of treatment of the specific therapies. As no specifications regarding tumor response assessments are made within the scope of this noninterventional study (neither examination methods nor time points are scheduled), objectives based on tumor response assessments, such as PFS as per a predefined measure (i.e., RECIST criteria with a central review), are not suitable. Based on the results of previous investigations, treatment duration appears to

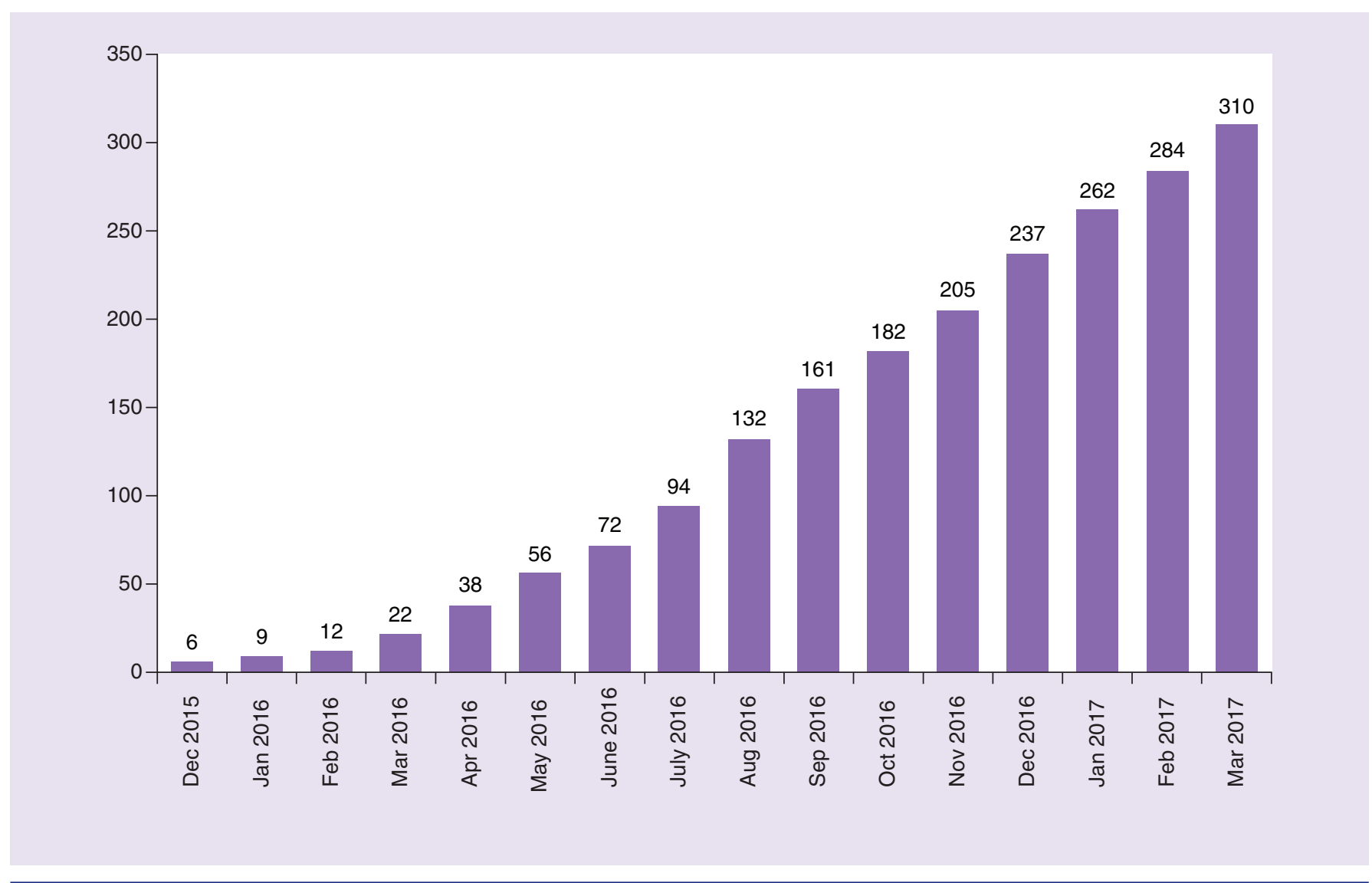

Figure 3. Numbers of patients included. Patients included in the documentation until 31 March 2017. 
be a practicable surrogate parameter for PFS in a routine setting [7]. However, the date of tumor progression as assessed by the investigator on the basis of local diagnostic standards is documented within the frame of the study. Further objectives include the assessment of:

- OS, defined as the time from start of therapy (start of pazopanib treatment in first line or start of everolimus therapy in third line) until the date of death due to any cause. For patients included in the third line, a second analysis of OS will be conducted where start of therapy equals the retrospectively documented start of nivolumab therapy in the second line;

- The effect of frequent lab analyses on frequency and severity of AEs (with focus on liver-related AEs), based on the number and time schedule of performed liver function tests and the number and severity of recorded liver-related AEs (descriptive analysis);

- Assessment of dose reductions/interruptions and treatment discontinuation as well as the evaluation of AEs with regard to concomitant medication;

- QoL with first-line pazopanib and second- and third-line everolimus as well as with secondline nivolumab, analyzed by evaluating the EQ-5D-5L questionnaire.

Prognostic factors related to the patient's global health status, such as Memorial SloanKettering Cancer Center prognostic score and Eastern Cooperative Oncology Group Performance Status, are documented if data are available. Subgroup analyses with regard to prognostic factors will be conducted if relevant patient numbers are reached for the respective subgroups.

\section{- Statistics}

Background and demographic variables will be presented descriptively. Quantitative variables will be presented by means of number of patients, mean, standard deviation, median, minimum and maximum. Categorical variables will be described by frequency and percentage values. OS will be represented by Kaplan-Meier curves. Four IAs are planned:

- The first IA will be performed in April 2017, 1 year after regulatory approval of nivolumab for mRCC in Europe. The aim is to observe, describe and analyze early trends regarding the use of nivolumab as a second-line treatment in Germany;

- The second IA will take place 1 year after accrual of the 200th patient (presumably by the end of 2017). The third and fourth IA will follow 1 year and 2 years later, respectively. The even temporal distribution of IAs over the course of the study allows to observe the growing data base in real-time and to adjust the study design and/or analyses to rising trends;

- The final analysis is planned to be performed in early 2021.

\section{Conclusion}

One of the currently recommended treatment options for patients with mRCC is first-line pazopanib followed by everolimus as a secondline treatment. However, data on the daily clinical use of these agents are not available, especially with regard to the different therapy sequences including pazopanib, everolimus and nivolumab. Furthermore, the treatment landscape for patients with mRCC has changed considerably during the last years, as nivolumab, cabozantinib and lenvatinib in combination with everolimus have been recently approved for the treatment of mRCC patients in Europe. Real-world data are needed to further evaluate the safety, tolerability, effectiveness and QoL of the various available treatment sequences in mRCC.

The prospective, noninterventional study, PAZOREAL was designed to evaluate the effectiveness, tolerability, safety and QoL in patients with mRCC starting either on pazopanib as first-line therapy or third-line everolimus plus/ minus lenvatinib following nivolumab. It allows collection of data on the clinical routine use of nivolumab as a second-line agent since the time the drug has gained EMA approval for the treatment of mRCC. Furthermore, in the course of the follow-up documentation, first basic data on the real-world use of the recently approved agents cabozantinib and lenvatinib can be obtained.

\section{Trial registration}

This study was registered at the German Health Authority's (Bundesinstitut fuer Arzneimittel und Medizinprodukte) database for noninterventional studies [20], NIS number 6687. 
Financial \& competing interests disclosure This study is sponsored by Novartis Pharma GmbH, Nuernberg, Germany. PJ Goebell received honoraria and advisory fees from Astellas, Bayer Healthcare, Bristol-Myer Squibb, Ipsen, Janssen, Lilly, Novartis, Pfizer, Roche and Sanofi. C Doehn received consultant fees and contract renumeration from Amgen, Bayer Healthcare, Bristol-Myers Squibb, GlaxoSmithKline, Novartis, Pfizer and Roche; he received contract fees from Ipsen as well as consultant fees from Eisai and holds stocks or stock options of AstraZeneca, Bayer and Bristol-Myers Squibb. C Grüllich received consultant fees from Novartis. V Grünwald received honoraria and advisory fees from Bristol-Myer Squibb, Ipsen, Novartis, Pfizer and Roche and honoraria from Eisai and Roche. T Steiner has no conflicts of interest to declare. $R$ Ehneß is an employee of Novartis Pharma GmbH, Germany, and holds stocks or stock options of Novartis and GlaxoSmithKline. M Welslau received Advisory Board fees, as well as for consulting and expert activities from different companies which are: Amgen, Astellas, AstraZeneca, Bristol-Myers-Squibb, Celgene, Gilead Sciences, Hexal, Janssen, Eli Lilly, medac, Novartis, Roche and Sanofi. The authors have no other relevant affiliations or financial involvement with any organization or entity with a financial interest in or financial conflict with the subject matter or materials discussed in the manuscript apart from those disclosed.

Writing assistance was provided by A Resch, Freelance Clinical Research Manager on behalf of Novartis Pharma GmbH, Nuernberg, Germany.

\section{Open access}

This work is licensed under the AttributionNonCommercial-NoDerivatives 4.0 Unported License. To view a copy of this license, visit http://creativecommons.org/ licenses/by-nc-nd/4.0/

\section{EXECUTIVE SUMMARY}

\section{Background}

- Real-world data are limited for the currently recommended therapy sequences with first-line pazopanib, followed by everolimus or other targeted therapies in the second line. With the approvals of new agents, the treatment algorithms for patients with metastatic renal cell carcinoma (mRCC) have changed markedly in recent years.

\section{The PAZOREAL study}

- The aim of this noninterventional study is to evaluate the effectiveness, tolerability, safety and quality of life in patients with mRCC treated with first-line pazopanib, second-line nivolumab or everolimus or third-line everolimus after nivolumab.

\section{Objectives}

- The PAZOREAL study is expected to provide invaluable data on the daily clinical use of available mRCC treatment options in different lines of therapy by observing the evolving treatment landscape in real time. Treatment and dosing parameters (dose reduction/interruption rates and reasons), disease assessments (time on drug for all lines of therapy), adverse events (AEs) with a focus on the effect of lab analyses frequency on incidence and severity of AEs with particular emphasis on liver AEs, follow-up treatments and overall survival are documented in $450 \mathrm{mRCC}$ patients in Germany.

\section{References}

Papers of special note have been highlighted as: - of interest; $\bullet$ of considerable interest

1 Protzel C, Maruschke M, Hakenberg OW. Epidemiology, aetiology, and pathogenesis of renal cell carinoma. Eur. Urol. 11(3), 52-59 (2012).

2 National Comprehensive Cancer Network, Inc. 2016 (Version 2.2017). www.nccn.org/professionals/

3 Escudier B, Porta C, Schmidinger M et al. Renal cell carcinoma: ESMO Clinical Practice Guidelines for diagnosis, treatment and follow-up. Ann. Oncol. 27(Suppl. 5), v58-v68 (2016).
4 Powles T, Staehler M, Ljungberg B et al. Updated EAU Guidelines for clear cell renal cancer patients who fail VEGF targeted therapy. Eur. Urol. 69(1), 4-6 (2016).

5 Sternberg CN, Davis ID, Mardiak J et al. Pazopanib in locally advanced or metastatic renal cell carcinoma: results of a randomized Phase III trial. J. Clin. Oncol. 28(6), 1061-1068 (2010).

-• Phase III trial with pazopanib versus placebo in treatment-naive and cytokinepretreated advanced renal cell carcinoma (RCC)/metastatic RCC (mRCC) patients demonstrating significant improvement in progression-free survival and tumor response compared with placebo.

6 Motzer RJ, Escudier B, Oudard S et al. Phase III trial of everolimus for metastatic renal cell carcinoma: final results and analysis of prognostic factors. Cancer 116(18), 4256-4265 (2010).

- Phase III trial with everolimus versus placebo in mRCC patients progressing on sunitinib and/or sorafenib which showed a prolonged progression-free survival with everolimus over placebo and established efficacy and safety of everolimus.

7 Motzer RJ, Hutson TE, Cella D et al. Pazopanib versus sunitinib in metastatic renal 
cell carcinoma. N. Engl. J. Med. 369, 722-731 (2013).

- Phase III head-to-head comparison of pazopanib versus sunitinib in previously untreated mRCC patients, showing non-inferiority of pazopanib.

8 Motzer RJ, Escudier B, McDermott DF et al. Nivolumab versus everolimus in advanced renal-cell carcinoma. N. Engl. J. Med. 373, 1803-1813 (2015).

- Phase III trial with nivolumab versus everolimus in previously treated patients with advanced RCC showing significant overall survival advantage and ORR improvement for nivolumab.

9 SmPC (German "Fachinformation") of Nivolumab (Opdivo ${ }^{\circledR}$ ), Bristol-Myers Squibb GmbH \& Co. KGaA, Munich, Germany (2016).

10 SmPC (German Fachinformation) of Cabozantinib (Cabometyx ${ }^{\mathrm{TM}}$ ), Ipsen Pharma GmbH, Ettlingen, Germany (2016).

11 SmPC (German Fachinformation) of Lenvatinib (Kisplyx $\left.{ }^{\circledast}\right)$, Eisai Europe Ltd. Hertfordshire, United Kingdom (2016).

12 Choueiri TK, Escudier B, Powles T et al. Cabozantinib versus everolimus in advanced renal-cell carcinoma. N. Engl. J. Med. 373, 1814-1823 (2015).

13 Choueiri TK, Escudier B, Powles T et al. Cabozantinib versus everolimus in advanced renal cellcarcinoma (METEOR): final results from a randomised, openlabel, Phase 3 trial. Lancet Oncol. 17, 917-927 (2016).

- PhaselII trial with cabozantinib versus everolimus in patients who progressed afterVEGFR-targeted therapy demonstrating a longer PFS and improved overall response rate (ORR) with cabozantinib.

14 Motzer RJ, Hutson TE, Glen H et al. Lenvatinib,everolimus, and the combination in patients with metastatic renal cellcarcinoma: a randomised, Phase 2, open-label, multicentre trial. Lancet Oncol. 16, 1473-1482 (2015).

- Phase II trialwith lenvatinib, everolimus and lenvatinib + everolimus as secondlinetreatment in mRCC patients demonstrating that lenvatinib + everolimus andlenvatinib alone resulted in a PFS benefit.

15 GermanS3 Guidelines: Leitlinienprogramm Onkologie (Deutsche

Krebsgesellschaft,Deutsche Krebshilfe, AWMF): Diagnostik, Therapie und Nachsorge desNierenzellkarzinoms, Kurzversion 1.0, 2015, AWMF Registernummer: 043/017OL. http://leitlinienprogramm-onkologie

16 Doehn C, Grünwald V, Steiner T et al. The diagnosis, treatment, and follow-up of renal cellcarcinoma. DtschArztebl Int. 113(35-36), 590-596 (2016).
17 Personal communication with Prof. Christian Doehn, S3 Guidelines Chair and Coordinator; 23 January 2017.

18 MarschnerN, Staehler M, Müller L et al. (2016). Survival of patients with advanced or metastatic renalcell carcinoma in routine practice differs from that in clinical trials analyses from the German Clinical RCC Registry. Clin. Genitourin. Cancer doi:10.1016/j.clgc.2016.08.022 (2016) (Epub ahead of print).

-• Amulticenter German cohort study assessing the trial eligibility of patientswith advanced/ metastatic RCC in routine practice and comparing the survivalof 'trial-ineligible' and potentially 'trial-eligible' patients. It was shownthat almost $60 \%$ of mRCC patients in German routine practice would be ineligiblefor participation in clinical trials and that first-line PFS and OS wereshorter for these patients as compared to 'trial-eligible' patients.

19 Marschner N, Müller L, Münch A et al. Adverse reactions in mRCC patients documented in routinepractice by German office-based oncologists and uro-oncologists. J. Oncol. Pharm. Practice doi:10.1177/1078155216632379 (2016) (Epub ahead of print).

20 Bundesinstitut fuer Arzneimittel und Medizinprodukte. http://awbdb.bfarm.de 